\title{
APPROXIMATELY DIFFERENTIABLE FUNCTIONS: THE $r$ TOPOLOGY
}

\author{
RICHARD J. O'MALLEY
}

It is shown that the coarsest topology making all approximately differentiable functions continuous is not the density topology. The correct topology, the $r$ topology, is introduced, and the structure of the open sets in this topology is examined. Among other things, it is proven that any $r$-open set must have nonempty Euclidean interior.

In the development of the $r$ topology, two new classes of functions play a role. These classes are the Baire $* 1$ approximately continuous functions and the ambivalent approximately continuous functions. For either class, $r$ is also the coarsest topology for which they are continuous.

1. Introduction. In this paper we examine functions $f:[0,1] \rightarrow$ $R$ which possess a finite approximate derivative everywhere in $[0,1]$. These functions are properly contained in the class of approximately continuous functions. In their study [4] of approximately continuous transformations, Goffman and Waterman present a topology which they label the density topology $d$. They show that with respect to $d$ the approximately continuous functions are continuous. For any collection of real-valued functions there is a coarsest topology relative to which each function in the collection is continuous. Goffman, Neugebauer and Nishiura [3] established that the coarsest such topology for the approximately continuous functions is precisely $d$. The connection between approximately differentiable functions and the density topology is clear. It presents no difficulty to show that the differentiable functions relative to $d$ are exactly the approximately differentiable functions. Thus it would appear that the density topology is the natural tool with which to examine the approximate behavior of functions. However, in this paper an unexpected fact surfaces. The density topology is not the coarsest topology making the approximately derivable functions continuous. Here we present the proper topology which we lable the $r$ topology. Besides being coarser than $d$ the $r$ topology is shown to possess several properties not common to $d$. For example, any set open in $r$ must have nonempty Euclidean interior.

In the development of the $r$ topology two new classes of functions play a role. These classes are the Baire ${ }^{*} 1$ approximately continuous functions and the ambivalent approximately continuous functions. For either class, $r$ is also the coarsest topology for which they are continuous. 
2. Preliminary theorems and definitions. All functions will be real-valued and defined on the interval $[0,1]$. When a function $f$ is restricted to a set $A$ we use the notation $f \mid A$. If we say that $f \mid A$ is continuous we will always mean relative to $A$. The symbol $|\cdot|$ will denote Lebesgue measure. It will be necessary to consider simultaneously various topological concepts such as closure (cl), or interior (int), with respect to several different topologies. For this reason we have adopted the convention of preceding each such idea with the symbol for the topology. When no prefix appears it should be assumed that the Euclidean topology is meant. For example, we will denote the interior of a set $A$ in the $d$ topology as $d$-int $(A)$. Finally, it will be essential to consider unions and intersections over various indexing sets. Whenever no confusion will result, the indexing set will not be explicitly mentioned. For example, we will use the notation $\cup F_{n}$ rather than usual $\bigcup_{n=1}^{\infty} F_{n}$.

Definition 2.1. Let $A$ be a measurable subset of $[0,1]$. The upper metric density of $A$ at a point $x$ is

$$
\lim _{n \rightarrow+\infty} \sup \left\{\frac{|A \cap I|}{|I|}:|I|<\frac{1}{n}\right\},
$$

where $I$ is any interval containing $x$. The lower metric density of $A$ at $x$ is defined similarly. When the upper and lower metric densities of $A$ at $x$ are equal their common value is called the density of $A$. at $x$.

DEFINITION 2.2. A measurable function is approximately continuous if for every $a<b$ the density of the set $\{x: a<f(x)<b\}$ is 1 at all of its points.

An approximately continuous function is of Baire class 1 and possesses the Darboux property [4].

For approximate differentiability the usual definition [6] is the following.

Definition 2.3.a. At a point $x_{0}$ a measurable function $f$ has a finite approximate derivative, $f_{a p}^{\prime}\left(x_{0}\right)$, if for every $\varepsilon>0$ the density of the set

$$
\left\{x:\left|\frac{f(x)-f\left(x_{0}\right)}{x-x_{0}}-f_{a p}^{\prime}\left(x_{0}\right)\right|<\varepsilon\right\}
$$

equals 1 at $x_{0}$. A measurable function is approximately differentiable if it has a finite approximate derivative at every point of $[0,1]$.

We note that every approximately differentiable function is approximately continuous. 
A definition equivalent to Definition 2.3.a will also be necessary later in the paper.

DeFinition 2.3.b. At a point $x_{0}$ a measurable function $f$ has a finite approximate derivative $f_{a p}^{\prime}\left(x_{0}\right)$ if there is a set $E$ having density 1 at $x_{0}$ such that, when $x$ is restricted to $E$,

$$
\lim _{x \rightarrow x_{0}} \frac{f(x)-f\left(x_{0}\right)}{x-x_{0}}=f_{a p}^{\prime}\left(x_{0}\right) .
$$

In [4] Goffman and Waterman defined the open sets of the density topology as follows:

Definition 2.4. A set $U$ is $d$-open if $U$ is measurable and has density 1 at all its points.

From this definition and the Lebesgue density theorem the following facts can be proven for any measurable set $U$.

REMARK 1. The $d$-interior of $U, d$-int $(U)$, consists precisely of those points of $U$ at which $U$ has density 1 .

REMark 2. The $d$-closure of $U, d$-cl $(U)$, consists of $U$ together with those points at which $U$ has positive upper metric density.

REMark 3. $|d-\operatorname{int}(U)|=|U|=|d-\operatorname{cl}(U)|$.

As mentioned in $\S 1$, Goffman, Neugebauer and Nishiura [3] established that $d$ is the coarsest topology for which the approximately continuous functions become continuous. An essential step in their proof is the so-called Lusin-Menchoff theorem. In this paper that theorem and its proof will be used in two different ways. We state the theorem in topological terms below. The proof given here is a slightly modified version of that in [3].

L-M THEOREM. Let $X$ be a closed set and $U$ a d-open set containing $X$. Then there is a closed set $P$ such that

$$
X \subset d \text {-int }(P) \subset P \subset U .
$$

Proof. For every natural number $n$ let

$$
R_{n}=\left\{x:(n+1)^{-1}<\delta(x, X) \leqq n^{-1}\right\} \cap U
$$

where

$$
\delta(x, X)=\inf \{|y-x|: y \in X\}
$$


Then $U=X \cup\left(\cup R_{n}\right)$. For every $n$ there is a closed set $P_{n} \subset R_{n}$ such that $\left|P_{n}\right|>\left|R_{n}\right|-2^{-n}$. Define $P=\left(\cup P_{n}\right) \cup X$. It is clear that $P$ is closed and $X \subset P \subset U$. In order to verify that $X \subset d$-int $(P)$ let $x$ belong to $X$. Let $\left\{I_{j}\right\}$ be a sequence of intervals such that $\cap I_{j}=\{x\}$ and $\left|I_{j}\right| \rightarrow 0$ as $j \rightarrow \infty$. For each $j$ let $n_{j}$ be the first integer larger than or equal to $\left|I_{j}\right|^{-1}-1$. Then by the definition of $R_{n}, I_{j} \cap R_{n}=$ $\varnothing$, for $n<n_{j}$. It follows from the definition of $P$ that

$$
\left|I_{j} \cap(U \backslash P)\right| \leqq \sum_{n \leqq n_{j}}\left|R_{n}\right| P_{n} \mid<2^{1-n_{j}}
$$

Thus

$$
\left|U \cap I_{j}\right| \leqq\left|P \cap I_{j}\right|+2^{1-n_{j}} .
$$

Since $n_{j}+1 \geqq I_{j}^{-1}$, we obtain

$$
\frac{\left|U \cap I_{j}\right|}{\left|I_{j}\right|} \leqq \frac{\left|P \cap I_{j}\right|}{\left|I_{j}\right|}+\left(n_{j}+1\right) 2^{1-n_{j}} .
$$

As $j \rightarrow+\infty$ we have $n_{j} \rightarrow+\infty$, so that

$$
1=\lim _{j \rightarrow+\infty} \frac{\left|U \cap I_{j}\right|}{\left|I_{j}\right|}=\lim _{j \rightarrow+\infty} \frac{\left|P \cap I_{j}\right|}{\left|I_{j}\right|} .
$$

This proves that $P$ has density 1 at $x$, so that, by Remark $1, x$ belongs to $d$-int $(P)$. This completes the proof.

We will also need the following corollary.

CoROLlary 2.1. Let $U$ be an $F_{o}$ d-open set. Then $U$ can be expressed as the union of closed sets $E_{n}$ with the property that $E_{n} \subset$ $d$-int $\left(E_{n+1}\right) \subset E_{n+1}$ for all $n$.

Proof. Since $U$ is an $F_{\sigma}$ set it can be expressed as the union of closed sets $F_{n}$. By the Lusin-Menchoff theorem for $F_{1}$ there is a closed set $P$ such that $F_{1} \subset d$-int $(P) \subset P \subset U$. Let $E_{1}=F_{1}$ and $E_{2}=$ $P$. Now assume that $E_{n+1}$ has been chosen so that

$$
E_{1} \subset d \text {-int }\left(E_{2}\right) \subset E_{2} \subset d \text {-int }\left(E_{3}\right) \subset \cdots \subset E_{n} \subset d \text {-int }\left(E_{n+1}\right) \subset E_{n+1} .
$$

Consider the set $E_{n+1} \cup F_{n+1}=H_{n+1}$. Then $H_{n+1}$ is a closed subset of $U$. Again an application of the Lusin-Menchoff theorem shows that there is a closed set $P$ such that $H_{n+1} \subset d$-int $(P) \subset P \subset U$. Let $E_{n+2}=P$.

It was mentioned in $\S 1$ that revelant to the study of approximately differentiable functions are the concepts of ambivalence and Baire ${ }^{*} 1$. We will need several results related to these two concepts.

Definition 2.5. A set $A$ is ambivalent if it is both an $F_{\sigma}$ set 
and a $G_{j}$ set. The property of being ambivalent is preserved under finite unions, finite intersections and finite complementations. All closed sets and all open sets are ambivalent.

Definition 2.6. A function $f$ is ambivalent if for each $a$ the sets $\{x: f(x)>a\}$ and $\{x: f(x)<a\}$ are ambivalent sets.

We note that an ambivalent function is of Baire class 1 , although the converse is not true.

Definition 2.7. A function $f$ is Baire * 1 if for every nonempty closed set $C$ there is an open interval $(a, b)$, with $(a, b) \cap C \neq \varnothing$, such that the restriction of $f$ to $C, f \mid C$, is continuous on $(a, b)$.

We note that any Baire $* 1$ function is of Baire class 1 .

The main properties of Baire * 1 functions possessing in addition the Darboux property are discussed in [5]. The following results are not in [5] and reveal the relationship between ambivalent functions and Baire * 1 functions. These results are necessary in this paper because approximately differentiable functions are Baire ${ }^{*} 1$ [7].

THeOREM 2.1. A function $f$ is Baire ${ }^{*} 1$ if and only if there is a sequence of closed sets $E_{n}$ such that $\cup E_{n}=[0,1]$ and $f \mid E_{n}$ is continuous for each $n$.

Proof. $\Leftrightarrow$ Let $K=\{x: f$ is continuous at $x\}$. From Definition 2.7 it follows that the interior of $K$ is a dense open set $V$. Let $J$ be the set of all those open intervals $I$ such that $I$ is the union of a sequence of closed sets $E_{n}$ with $f \mid E_{n}$ continuous for each $n$. Let $W=\mathrm{U} I$, the union being taken over all $I$ in $J$. Every component of $V$ is in $J$, so that $W$ is a dense open set. Moreover, every component of $W$ is itself in $J$. Thus $W$ itself has the property that $W$ is the union of a sequence of closed sets $E_{n}$ with $f \mid E_{n}$ continuous for each $n$. We need only show that $W=[0,1]$. Let $C=[0,1] \backslash W$. If $C \neq \varnothing$, then by Definition 2.7 there is an open interval $(a, b)$ with $(a, b) \cap C \neq \varnothing$ and $f \mid C$ continuous on $(a, b)$. However, $C \cap(a, b)$ is an $F_{a}$, so that $C \cap(a, b)$ is the union of closed sets $C_{n}$ with $f \mid C_{n}$ continuous. Since $(a, b) \backslash C$ is an open subset of $W$ it also is the union of closed sets $E_{n}$ with $f \mid E_{n}$ continuous. Thus $(a, b)$ is contained in $J$ and is a subset of $W$. This contradicts $(a, b) \cap C \neq \varnothing$.

$\Leftrightarrow$ This part of the proof needs only a simple application of the Baire category theorem.

THeOREM 2.2. If $f$ is Baire * 1 , then $f$ is ambivalent.

Proof. Let $a$ be given. Since $a$ set is ambivalent if and only 
if its complement is ambivalent, we need only show that $\{x: f(x) \leqq a\}$ and $\{x: f(x) \geqq a\}$ are ambivalent sets. We show this for $\{x: f(x) \leqq a\}$ only. Let $E_{n}$ be a sequence of closed sets such that $f \mid E_{n}$ is continuous and $\cup E_{n}=[0,1]$. Then $\{x: f(x) \leqq a\}=U\left[\{x: f(x) \leqq a\} \cap E_{n}\right]$. For each $n$ the set $E_{n} \cap\{z: f(x) \leqq a\}$ is a closed set. Thus $\{x: f(x) \leqq a\}$ is an $F_{\sigma}$. However, this set is also a $G_{\delta}$ because $f$ is Baire 1 .

THEOREM 2.3. Let $I$ be a closed interval $[a, b]$. Let $U$ be an ambivalent subset of I with $(a, b) \cap U \neq \varnothing$. Suppose in addition that every point of $(a, b) \cap U$ is a bilateral limit point of $U$. Then $U$ has nonempty interior.

Proof. Suppose $U$ contains no open interval $J$. Since both $U$ and $I \backslash U$ are $F_{\sigma}$ sets, an application of the Baire category theorem to $U \cup(I \backslash U)$ implies that int $(I \backslash U)=V$ is a dense open subset of I. Moreover, if $I \backslash U$ contains an interval $(c, d)$ with $a<c<d<b$, then it must also contains the endpoints $c$ and $d$. This is because every point of $(a, b) \cap U$ is a bilateral limit point of $U$. The set $I \backslash V$ is a nowhere dense perfect set. The set $U$ is a dense subset of $I \backslash V$ and also a $G_{\dot{\delta}}$. However, consider the endpoints of those components of $V$ contained in $(a, b)$. These points are in $I \backslash U$, and they form a dense subset of $I \backslash V$. Thus $(I \backslash V) \backslash U$ is a dense subset of $I \backslash V$. But $(I \backslash V) \backslash U$ is a $G_{\delta}$ set disjoint from $U$. This would yield two dense disjoint $G_{j}$ subsets of the perfect set $I \backslash V$, and contradict the Baire category theorem.

The rational numbers provide an example of the fact that the countable union of ambivalent sets need not be a $G_{\delta}$ set. However, we will have use for the following theorem.

THEOREM 2.4. Let $R_{n}$ be a sequence of ambivalent sets. Let $U_{n}$ be a sequence of pairwise disjoint open sets with $R_{n} \subset U_{n}$. Then $\cup R_{n}$ is an ambivalent set.

Proof. We need only show that $\cup R_{n}$ is a $G_{\dot{j}}$. For each $n$ there is a sequence of open sets $G_{n k}, k=1,2, \cdots$ such that $\bigcap_{k} G_{n k}=R_{n}$. Without loss of generality we may assume that $G_{n k} \subset U_{n}$ for all $n$ and $k$. For each fixed $k$ let the union over $n$ of $G_{n k}$ be denoted by $V_{k}$. Then $V_{k}$ is open for each $k$ and $\cap V_{k}=\bigcup R_{n}$.

The following theorem can be considered as a strong version of the fact that the Euclidean topology is normal. The proof is not difficult. It is given here as there seems to be no adequate reference.

THEOREM 2.5. Let $X$ and $Y$ be disjoint, nonempty, closed subsets of $[0,1]$. Then there is a differentiable function $g$ satisfying 
(1) $g(x)=1$ for all $x$ in $X$,

(2) $g(x)=0$ for all $x$ in $Y$,

(3) $0<g(x)<1$ for all $x$ in $[0,1] \backslash(X \cup Y)$, and

(4) $g^{\prime}(x)=0$ for all $x$ in $X \cup Y$.

Proof. Let $W=[0,1] \backslash(X \cup Y)$. Let the components of $W$ be arranged in a sequence $\left(a_{n}, b_{n}\right)$. We will assume that both 0 and 1 belong to $X \cup Y$. (In the other case, let $x_{0}$ and $x_{1}$ be the greatest lower bound and least upper bound of $X \cup Y$. Construct the function $g$ as below on the interval $\left[x_{0}, x_{1}\right]$, and extend appropriately to the interval $[0,1]$.) The endpoints of the intervals $\left(a_{n}, b_{n}\right)$ belong to $X \cup Y$. Only finitely many components can have one endpoint in $X$ and the other in $Y$. This is because the sets $X$ and $Y$ are disjoint and closed, so that inf $\{|x-y|: x$ belongs to $X, y$ belongs to $Y\}>0$. Let the sequence of intervals be rearranged so that $\left(a_{1}, b_{1}\right), \cdots,\left(a_{N}, b_{N}\right)$ are the components with left endpoint in $X$ and right endpoint in $Y$, and $\left(a_{N+1}, b_{N+1}\right), \cdots,\left(a_{N+K}, b_{N+K}\right)$ are those with right endpoint in $X$ and left endpoint in $Y$. For $i=1, \cdots, N$ we define $g$ on $\left[a_{i}, b_{i}\right]$ to be a strictly decreasing differentiable function with $g\left(a_{i}\right)=1, g\left(b_{i}\right)=$ 0 , and $g^{\prime}\left(a_{i}\right)=g^{\prime}\left(b_{i}\right)=0$. For $i=1, \cdots, K$ we define $g$ on $\left[a_{N+i}, b_{N+i}\right]$ to be a strictly increasing differentiable function with $g\left(a_{N+i}\right)=0$, $g\left(b_{N+i}\right)=1$, and $g^{\prime}\left(a_{N+i}\right)=g^{\prime}\left(b_{N+i}\right)=0$. Let $n>N+K$ be given. Let $g$ be defined on $\left[a_{n}, b_{n}\right]$ as a differentiable function having

(i ) $g^{\prime}\left(a_{n}\right)=g^{\prime}\left(b_{n}\right)=0$,

(ii) $\left|g^{\prime}(x)\right|<1 / n$, for all $x$ in $\left[\alpha_{n}, b_{n}\right]$,

(iii) $0<g(x)<1$, for all $x$ in $\left(a_{n}, b_{n}\right)$, and

(iv) $g\left(a_{n}\right)=g\left(b_{n}\right)=\left\{\begin{array}{l}0 \text { if } a_{n} \text { belongs to } Y, \\ 1 \text { if } \alpha_{n} \text { belongs to } X \text {. }\end{array}\right.$

Finally, let $g(x)=1$ for all $x$ in $X \backslash \cup\left[a_{n}, b_{n}\right]$ and 0 for all $x$ in $Y \backslash \cup$ $\left[a_{n}, b_{n}\right]$. It is not difficult to show that $g$ is differentiable and satisfies (1) through (4).

This completes the preliminary theorems.

3. The $r$ topology. Theorem 3.1 forms the cornerstone of this section. It reveals the relation between approximately differentiable functions and ambivalence.

THEOREM 3.1. Let $U$ be an ambivalent d-open set with $[0,1] \backslash U$ nonempty. Let $X_{0}$ be any closed subset of $U$. There is a function $g$ satisfying the following 6 properties.

(1) $g(x)$ is upper-semicontinuous.

(2) $g(x)=1$ for all $x$ in $X_{0}$.

(3) $g(x)$ is approximately differentiable for all $x$ in $U$.

(4) $0<g(x)<1$ for all $x$ in $U \backslash X_{0}$. 
(5) $g(x)=0$ for all $x$ in $[0,1] \backslash U$.

(6) $g$ is differentiable, with derivative zero, for all $x$ in $[0,1] \backslash U$.

(It should be noted that (3) and (6) together imply that $g$ is approximately differentiable.)

Proof. The set $[0,1] \backslash U$ is an $F_{\sigma}$. Express $[0,1] \backslash U$ as the union of a sequence of closed sets $Z_{n}$ with $Z_{n} \subset Z_{n+1}$ for all $n$. The set $U$ is an $F_{o} d$-open set. Using Corollary 2.1 we express $U$ as the union of closed sets $E_{n}$ with $E_{1}=X_{0}$ and $E_{n} \subset d$-int $\left(E_{n+1}\right)$ for all $n$. For each $n$, let $f_{n}$ be a differentiable function satisfying the four properties of Theorem 2.5 for $E_{n}=X$ and $Y=Z_{n}$. Let $g_{n}$ be the product of $f_{1}$ through $f_{n}$. For each $n, g_{n}(x)$ is a differentiable nonnegative function. For each $x$, the sequence $g_{n}(x)$ is a nonincreasing sequence of nonnegative numbers. Hence the pointwise limit of the sequence $g_{n}(x)$ exists. This pointwise limit is upper-semicontinuous. We label it $g(x)$ and show that the other five conditions are satisfied by $g$.

Proof of (2). Let $x$ belong to $X_{0}$. Then $x$ belongs to $E_{n}$ for all $n$. By the choice of $f_{n}$ we have $f_{n}(x)=1$ for all $n$. Hence $g_{n}(x)=$ $f_{1}(x) \cdot f_{2}(x) \cdot \cdots \cdot f_{n}(x)=1$ for all $n$, and $g(x)=1$.

Proof of (3) and (4). Let $x_{0}$ belong to $U$. Let $N$ be the first index for which $x_{0}$ belongs to $E_{N}$. For all $x$ in $E_{N+1}$ and $n>N$ we have $f_{n}(x)=1$. Hence $g(x)=g_{N}(x)$ for all $x$ in $E_{N+1}$. Since the function $g_{N}(x)$ is differentiable, when $x$ is restricted to $E_{N+1}$ we have

$$
\lim _{x \rightarrow x_{0}} \frac{g(x)-g\left(x_{0}\right)}{x-x_{0}}=\lim _{x \rightarrow x_{0}} \frac{g_{N}(x)-g_{N}\left(x_{0}\right)}{x-x_{0}}=g_{N}^{\prime}\left(x_{0}\right) .
$$

Since $x_{0} \in d$-int $\left(E_{N+1}\right), E_{N+1}$ has density 1 at $x_{0}$. Therefore, by Definition 2.3.b, $g$ has an approximate derivative $g_{a p}^{\prime}\left(x_{0}\right)=g_{N}^{\prime}\left(x_{0}\right)$. If, in addition, $x_{0}$ belongs to $U \backslash X_{0}$ then $N>1$ and $g\left(x_{0}\right)=g_{N+1}\left(x_{0}\right)=f_{1}\left(x_{0}\right) \cdots \cdots f_{N-1}\left(x_{0}\right)$. But $0<f_{i}\left(x_{0}\right)<1$ for $i=1,2, \cdots, N-1$ by Condition 3 of Theorem 2.4, so that $0<g\left(x_{0}\right)<1$.

Proof of (5) and (6). Let $x_{0}$ belong to $[0,1] \backslash U$. Let $N$ be the first index with $x_{0}$ in $Z_{N}$. Then $f_{N}\left(x_{0}\right)=0=g_{N}\left(x_{0}\right)=g\left(x_{0}\right)$. For all $x, g_{N}(x)$ is a differentiable function. If $N=1$ then $g_{N}^{\prime}\left(x_{0}\right)=f_{1}^{\prime}\left(x_{0}\right)=0$. If $N>1$ then $g_{N}^{\prime}\left(x_{0}\right)=g_{N-1}\left(x_{0}\right) \cdot f_{N}^{\prime}\left(x_{0}\right)+g_{N-1}^{\prime}\left(x_{0}\right) \cdot f_{N}\left(x_{0}\right)$. Both $f_{N}^{\prime}\left(x_{0}\right)$ and $f_{N}\left(x_{0}\right)$ equal zero. Hence

$$
\lim _{x \rightarrow x_{0}} \frac{g_{N}(x)-g_{N}\left(x_{0}\right)}{x-x_{0}}=\lim _{x \rightarrow x_{0}} \frac{g_{N}(x)}{\left|x-x_{0}\right|}=0 .
$$


Since $0 \leqq g(x) \leqq g_{N}(x)$ for all $x$, we have

$$
\lim _{x \rightarrow x_{0}} \frac{g(x)}{\left|x-x_{0}\right|}=0=\lim _{x \rightarrow x_{0}} \frac{g(x)-g\left(x_{0}\right)}{x-x_{0}}=g^{\prime}\left(x_{0}\right) .
$$

Definition 3.1. Let $B$ be the family of all sets which are ambivalent and $d$-open.

DEFINITION 3.2. Let the coarsest topology making the approximately differentiable functiable continuous be denoted by $r$.

THEOREM 3.2. The family $B$ forms a basis for $r$. Further, $r$ is the coarsest topology for which either the Baire * 1 approximately continuous or ambivalent approximately continuous functions are continuous functions are continuous.

Proof. Let $S$ be any collection of real-valued functions. If we let $f$ vary over the functions in $S$ and let $a$ vary through the real numbers, the resultant family of sets $\{x: f(x)>a\},\{x: f(x)<a\}$ forms a subbasis for the coarsest topology making each function in $S$ continuous.

Now in the case of approximately differentiable functions $f$ we have that both $-f$ and $f+a$ are also approximately differentiable. This yields that a subbasis for the $r$-topology is the family $Q$ of sets $U=\{x: f(x)>0\}$ for some approximately differentiable $f$. Each such $U$ is $d$-open because $f$ is approximately continuous. Further, $U$ is ambivalent because an approximately differentiable function is Baire * 1, and Baire * 1 functions are ambivalent by Theorem 2.2. Thus $Q \subset B$. In addition, Theorem 3.1 guarantees that every $d$-open ambivalent set is contained in $Q$. Thus $Q=B$. By noting that the family $B$ is closed under finite intersections, we have that $B$ is actually a basis for $r$.

For the second part of the theorem we note that the approximately differentiable, Baire ${ }^{*} 1$ approximately continuous and ambivalent approximately continuous functions form three increasing classes of functions. The coarsest topology, $r_{1}$, making approximately continuous Baire ${ }^{*} 1$ functions continuous will contain the $r$-topology and be contained in the coarsest topology, $r_{2}$, making approximately continuous ambivalent functions continuous. However, as above, a subbasis for $r_{2}$ will be the family of sets $W=\{U=\{x: f(x)>0\}$ for some ambivalent approximately continuous $f\}$. Each such $U$ is $d$-open and ambivalent. Thus $W=B$. Thus $r, r_{1}$, and $r_{2}$ are generated by the same basis and are identical.

For precision we give the following definition. 
Definition 3.3. A set $U$ is $r$-open if $U$ is the union of sets $V$ from the family $B$. A set $U$ is called an $r$-basis set if $U$ belongs to $B$.

It is clear that any $r$-open set is $d$-open and hence measurable. It would be noteworthy if, in addition, each $r$-open set were ambivalent. That such is not the case will be shown. First we examine more closely the structure of $r$-open sets.

THEOREM 3.3. If $U$ is r-open, then $U$ contains an open interval in any one-sided neighborhood of any of its points.

Proof. Let $x$ belong to $U$. There is an $r$-basis set $W(x)$ with $\{x\} \subset W(x) \subset U$. Let $I=[a, b]$ be any closed interval having $x$ as one endpoint. Then since $W(x)$ has density 1 at all its points, every point of $(a, b) \cap W(x)$ is a bilateral limit point of $W(x)$. Since $W(x) \cap I$ is ambivalent, Theorem 2.3 guarantees that $W(x) \cap I$ has nonempty interior.

COROLLARY 3.1. The $r$ topology is strictly coarser than the density topology.

Proof. Let $X$ be any measurable set with empty interior and positive measure. Let $X_{0}=d$-int $(X)$. By Remark 3 of $\S 1,\left|X_{0}\right|=$ $|X|>0$. The set $X_{0}$ is $d$-open but not $r$-open. In fact, $r$-int $\left(X_{0}\right)=$ $\varnothing$ because of Theorem 2.3.

Corollary 3.2. A set $A$ is dense (nowhere dense) in the $r$ topology if and only if it is dense (nowhere dense) in the Euclidean topology.

Proof. It will suffice to show that if a set $A$ is dense in an open interval $I$ then $A$ is $r$-dense in $I$. Let $W$ be any $r$-open set contained in $I$. Then $W$ has nonempty Euclidean interior $Q$. Then $Q \cap A \neq \varnothing$ because $A$ is dense in $I$. Hence $A \cap W \neq \varnothing$, and $A$ is $r$-dense in $I$.

Corollary 3.2 implies that $[0,1]$ with the $r$ topology is a space in which Blumberg's theorem [1] holds. This is not true of $[0,1]$ with the $d$ topology [7].

Corollary 3.3. For any set $A, \operatorname{cl}(A) \backslash r-\operatorname{cl}(A)$ is a nowhere dense set.

An application of Theorem 3.2 to $r$-continuous functions leads to: 
CoROLLARY 3.4. Let $f$ be r-continuous and $\{x: a<f(x)<b\} \neq \varnothing$. Then $\{x: a<f(x)<b\}$ has nonempty interior.

CoROLLARY 3.5. Let $f$ be r-continuous and $I$ any closed subinterval of $[0,1]$. Let $C=\{x: f$ is continuous at $x\} \cap I$. Then the image of $C$ is dense in the image of $I$.

Proof. It need only be noted that all $r$-continuous functions are approximately continuous and hence Baire class 1 .

CoROLlaRy 3.6. Let $f$ be r-continuous. If $\{x: f(x)=a\}$ is dense in $[0,1]$ then $\{x: f(x)=a\}=[0,1]$.

CoRollary 3.7. If $U$ is r-open and $V=\operatorname{int}(U)$, then $r$-cl $(U)=$ $r-\operatorname{cl}(V)$.

Proof. Let $x$ be any $r$-limit point of $U$. Let $W$ be any $r$-open set containing $x$. Then $W \cap U$ is a nonempty $r$-open set. By Theorem 3.3, $W \cap U$ has nonempty interior $Q$. Now $Q \subset V$, so that

$$
(W \cap V) \backslash\{x\} \neq \varnothing,
$$

and $x$ is an $r$-limit point of $V$.

It follows from Remark 2 of $\S 2$ that any set of measure zero has no $d$-limit point and hence is $d$-closed. The rationals show that this property does not carry over to the $r$ topology. However, we have the following corollary which will be improved after Theorem 3.5. We present this corollary now, as it is needed as a foundation for discussion of the normality of the $r$ topology.

Proposition 3.1. If $X$ is closed and $|X|=0$, then $X$ has no $r$-limit point. (Any subset of $X$ is $r$-closed.)

Proof. Let $x$ belong to $[0,1]$ and consider $[0,1] \backslash X=U$. Then $U$ is an open set with $|U|=1$. Hence $U \cup\{x\}$ is an $r$-basis set $V$, with $[V \cap X] \backslash\{x\}=\varnothing$.

Proposition 3.2. There is an r-open set which is not an ambivalent set.

Proof. Let $C$ be the Cantor set. Let $U=[0,1] \backslash C$. Let $S=$ $\left\{x_{n}, n=1,2, \cdots\right\}$ be any countable dense subset of $C$. For each $n$, $U \cup\left\{x_{n}\right\}$ an $r$-basis subset of $[0,1]$. Hence $S \cup U$ is $r$-open. However, $S \cup U$ would be ambivalent if and only if $S$ were ambivalent. $S$ cannot be a $G_{\dot{\delta}}$, however. 
THEOREM 3.4. The $r$ topology is not normal.

Proof. Let $X$ and $Y$ be any two disjoint dense subsets of the Cantor set. By Proposition 3.1, both $X$ and $Y$ are $r$-closed. There can be no $r$-continuous function $f$ which is 1 on $X$ and 0 on $Y$, because all $r$-continuous functions are approximately continuous and hence Baire 1. As Baire 1 functions, any $r$-continuous function must have a point of relative continuity in any closed set.

The next series of theorems will be used to establish precisely how close the $r$ topology comes to being normal. In the process, an analogue of the Lusin-Menchoff theorem is given in two stages.

THEOREM 3.5. If $U$ is r-open, there exists a countable collection of sets from $B, F_{n}$, with $\cup F_{n} \subset U$ and $\left|\cup F_{n}\right|=|U|$.

Proof. Let $B(U)$ be the collection of sets from $B$ which are contained in $U$. Let $\Omega$ be the collection of all sets which are the union of a countable subfamily of sets from $B(U)$. Let

$$
a=\sup \{|H|: H \in \Omega\} \text {. }
$$

Obviously, $\alpha \leqq|U|$. Further, there is a set $H$ from $\Omega$ such that $|H|=a$. Let $F_{n}, n=1,2, \cdots$, be the countable subfamily from $B(U)$ with $H=\bigcup F_{n}$. Suppose $\left|U \backslash \cup F_{n}\right|>0$. Then by Remarks 1 and 3 of $\S 2$ there is a point $x$ from $U$ at which $U \backslash \cup F_{n}$ has density 1 . Since $x$ belongs to $U$ there is a set $F(x)$ from $B$ with $\{x\} \subset F(x) \subset U$. Since $F(x)$ has density 1 at $x$ and $\cup F_{n}$ has density zero at $x$, we have $\left|F(x) \backslash \cup F_{n}\right|>0$. Hence $|F(x) \cup H|>|H|=\alpha$. However, $F(x) \cup$ $H$ belongs to $\Omega$. This contradiction implies that $\left|\cup F_{n}\right|=|U|$.

THEOREM 3.6 (L-M 1). Let $U$ be r-open and $X$ a closed subset of $U$. Then there is an r-basis set $E$ with $X \subset E \subset U$.

Proof. Let $R_{n}$ be defined as in the Lusin-Menchoff theorem of $\S 2$. Let $V_{n}=\left\{x:(n+1)^{-1}<\delta(x, X)<n^{-1}\right\}$. The sets $V_{n}$ are open because $\delta(x, X)$ is a continuous function. Further $R_{n} \backslash V_{n}$ is a finite set for each $n$. Thus $\cup V_{n} \cup X$ has density 1 at all its points. For each $n, U_{n}=V_{n} \cap U$ is an $r$-open set. Let $n$ be fixed. By Theorem 3.5 there is a sequence of $r$-basis sets $F_{n k}$ with $\left|U_{k} F_{n k}\right|=\left|U_{n}\right|$. We select a finite $K(n)$ such that $\left|\bigcup_{1 \leqq K \leqq(n)} F_{n k}\right|>\left|U_{n}\right|-2^{-n}$. Then $\bigcup_{1 \leqq k \leqq K(n)} F_{n k}$ is an $r$-basis set $E_{n}$. Now we set $E=\bigcup E_{n} \cup X$. The set $U E_{n}$ is an ambivalent set with density 1 at all of its points. The set $U E_{n}$ is an ambivalent set because $E_{n} \subset V_{n}$ and $\left\{V_{n}\right\}$ forms a sequence of pairwise disjoint open sets. This implies that to show that $E$ is an $r$-basis set requires only that we establish that $E$ has 
density 1 at each point of $X$. The proof of this involves the same computations as those given in the L-M theorem of $\S 2$.

THEOREM 3.7. Let $U$ be an r-open set and $x_{0}$ a point at which $U$ has density 1 . Then $U \cup\left\{x_{0}\right\}$ is r-open.

Proof. Let $U_{n}=\left\{x:(n+1)^{-1}<\left|x-x_{0}\right|<n^{-1}\right\} \cap U . \quad$ As in the proof of Theorem 3.6 we proceed to find an $r$-basis set $E_{n} \subset U_{n}$ with $\left|E_{n}\right|>\left|U_{n}\right|-2^{-n}$. Then $\cup E_{n} \cup\left\{x_{0}\right\}$ is an $r$-basis set contained in $U \cup\left\{x_{0}\right\}$.

Theorem 3.7 leads us to a characterization of which $d$-open sets are $r$-open.

THEOREM 2.8. A set $U$ is r-open iff $U$ is d-open and there is a sequence of $r$-basis sets $F_{n}$ with $F_{n} \subset U$ for each $n$ and $\left|\cup F_{n}\right|=|U|$.

Proof. $(\Longrightarrow)$ This is merely Theorem 3.5.

$\left(\Longleftarrow\right.$ Let $V=\bigcup F_{n}$. Then $V$ is $r$-open and $|V|=|U|$. Let $x$ belong to $U \backslash V$. The set $V$ has density 1 at $x$ since $|U|=|V|$. Therefore, Theorem 3.7 guarantees that $V \cup\{x\}$ is $r$-open. Thus $U=\bigcup\{V \cup\{x\}: x$ belongs to $U \backslash V\}$ is $r$-open.

Corollary 3.8. Let $U$ be r-open and $V=d$-cl $(U)$. Then $d$-int $(V)=$ $r$-int $(V)$.

Proof. By Remarks 1 and 3 of $\S 2$ we have $|V|=|U|, U \subset V$, and $d$-int $(V)=\{x \in V: V$ has density 1 at $x\}=\{x \in V: U$ has density 1 at $x\}$. If $x$ belongs to $d$-int $(V)$ then by Theorem 3.7, $U \cup\{x\}$ is $r$-open. Hence $x$ belongs to $r$-int $(V)$. The other inclusion is true since for any set $A, r$-int $(A) \subset d$-int $(A)$.

We now improve Proposition 3.1.

Proposition 3.3. Let $X$ be r-closed and $|X|=0$. Then $X$ has no r-limit points.

Proof. Let $x \in[0,1]$ and $U=[0,1] \backslash X$. Then $U$ is $r$-open, and $U$ has density 1 at $x$. Hence $U \cup\{x\}$ is $r$-open, and $x$ is not an $r$-limit point of $X$.

Finally, in comparison with Theorem 3.4 we have that the $r$ topology is "near" normal in the following sense.

THEOREM 3.9. Let $X$ be a nonempty closed set and $Y$ a non- 
empty r-closed set with $X \cap Y=\varnothing$. There is an r-continuous function $g$ with

(a) $g(x)=1$, for $x$ in $X$,

(b) $0 \leqq g(x)<1$, for all $x$ in $[0,1] \backslash X$,

(c) $g(x)=0$, for all $x$ in $Y$, and

(d) $g(x)$ is upper-semicontinuous.

Proof. Let $W=[0,1] \backslash Y$. Then set $W$ is $r$-open and $X \subset W$. By Theorem 3.6 there is an $r$-basis set $U$ with $X \subset U \subset W$. By Theorem 3.1 there is an approximately differentiable function satisfying (a), (b), (c), and (d). Since, by Theorem 3.2, $g$ is $r$-continuous, we are finished.

COROLLARY 3.9. The $r$ topology is completely regular.

TheOREM 3.10, (L-M 2). Let $X$ be a closed set and $U$ an r-open set with $X \subset U$. Then there is a closed set $P$ with $X \subset r$-int $(P) \subset$ $P \subset U$.

Proof. Let $Y=[0,1] \backslash U$. If $Y=\varnothing$ then there is nothing to prove. If $Y \neq \varnothing$ then $Y$ is $r$-closed and disjoint from $X$. Let $g$ be the function described in Theorem 3.8. Then

$$
X=\{x: g(x)=1\} \subset\left\{x: g(x)>\frac{1}{2}\right\} \subset\left\{x: g(x) \geqq \frac{1}{2}\right\} \subset U .
$$

Since $g$ is upper-semicontinuous, $\{x: g(x) \geqq 1 / 2\}$ is closed. Let $P=$ $\{x: g(x) \geqq 1 / 2\}$.

Corollary 3.10. Let $U$ be an $F_{o}$ r-open set. Then $U$ can be expressed as the union of closed sets $E_{n}$ with the property that $E_{n} \subset r$-int $\left(E_{n+1}\right) \subset E_{n+1}$ for all $n$.

Proof. The proof is the same as that of Corollary 2.1, using Theorem 3.10 in place of the L-M theorem of $\S 2$.

We end the paper by pointing out several possible areas for further research.

A. Throughout the paper we have restricted our attention to the interval $[0,1]$. However, there is no obstacle to extending the $r$ topology to $R^{n}$ using for basis the collection of sets $B=\{U: U$ is ambivalent and $d$-open\}. Here there are several options as to the actual $d$ topology we choose because in [3] Goffman, Neugebauer and Nishiura have defined three different density topologies. 
B. There is a class of functions which are continuous relative to the $r$ topology which have not been mentioned up to this point. Namely, the collection of functions which are approximately continuous everywhere and continuous almost everywhere. To show this we prove:

THEOREM 3.11. If $f$ is approximately continuous everywhere and continuous almost everywhere, then $f$ is r-continuous.

Proof. Let $a$ be fixed and $\{x: f(x)>a\}=U \neq \varnothing$. Then $|U|>0$ because $f$ is approximately continuous, and int $(U)=V \neq \varnothing$ because $f$ is continuous at almost every point of $U$. Indeed $|U|=|V|$. Thus an application of Theorem 3.8 gives that $U$ is $r$-open. The set $\{x: f(x)<a\}$ is dealt with the same way.

One reason that this class of functions was not mentioned earlier is that $r$ is not the coarsest topology for which these functions become continuous.

Definition 3.4. A set $U$ is almost open if $U$ is $d$-open and $|U|=$ $|\operatorname{int}(U)|$.

THEOREM 3.12. The collection of almost open sets forms a topology which we label a.e.

Proof. There is only one part that is not immediate. Namely, if $I$ is any indexing set and for each $\alpha$ in $I U_{\alpha}$ is an almost open set, then $\left|\operatorname{int}\left(\cup U_{\alpha}\right)\right|=\left|\cup U_{\alpha}\right|$. To see this we proceed along the same lines as Theorem 3.4 to find a countable subfamily $\left\{\alpha_{n}\right\}$ of $I$ with $\left|\cup U_{\alpha_{n}}\right|=\left|\cup U_{\alpha}\right|$. Then $\left|\operatorname{int}\left(\cup U_{\alpha_{n}}\right)\right|=\left|\cup U_{\alpha}\right|$.

COROLlARY 3.11. If $f$ is approximately continuous everywhere and continuous almost everywhere, then $f$ is continuous with respect to the a.e. topology.

Proof. As in the proof of Theorem 3.11 it is clear that for each fixed a both $\{x: f(x)>a\}$ and $\{x: f(x)<a\}$ are $d$-open and have Euclidean interiors equal to their respective measures.

THEOREM 3.13. The a.e. topology is coarser than the $r$ topology.

Proof. Let $X$ be a Cantor set of positive measure. Let $[0,1] \backslash X=$ $U$. From each component of $U$ delete the midpoint. Call the new open set thus obtained $V$. Let $W=V \cup X$. Then $W$ is an $r$-basis set. To see this note first $|W|=1$, so this $W$ is $d$-open. Next, since both open and closed sets are ambivalent and the union of two 
ambivalent is ambivalent, $W$ is an $r$-basis set. Finally, $\operatorname{int}(V \cup X)=$ $V$, and $|V|<|W|$ so that $W$ is not a.e. open.

ACKNOWLEDGMENTs. (1) The author would like to express his gratitude to Prof. Clifford Weil. His suggestions and support throughout the development of the paper were greatly appreciated.

(2) It has come the the author's attention that the functions which we labeled Baire* 1 were considered in a paper, [8], by H. W. Ellis. In that paper Ellis labeled such functions [CG].

\section{REFERENCES}

1. H. Blumberg, New properties of all real functions, Trans. Amer. Math. Soc., 24 (1922), 113-128.

2. H. W. Ellis, Darboux properties and applications to nonabsolutely convergent integrals, Canad. Math. J., 3 (1951), 471-484.

3. C. Goffman, C. J. Neugebauer and T. Nishiura, Density topology and approximate continuity, Duke Math. J. (1961), 497-505.

4. C. Goffman and D. Waterman, Approximately continuous transformations, Proc. Amer. Math. Soc., 12 (1961), pp. 116-121.

5. R. J. O'Malley, Baire * 1, Darboux functions, Proc. Amer. Math. Soc., 60 (1976), 187-192.

6. S. Saks, Theory of the integral, Monographie Matematyczne, 7, Warsawa-Lwów, 1937.

7. G. Tolstoff, Sur la dérivé approximative exacte, Mat. Sb., 4 (1938), 499-504.

8. H. E. White, Jr., An application of a theorem by R. E. Zink, Notices Amer. Math. Soc. Abstract 76T-B135, 23, 5 A487.

Received October 15, 1976 and in revised form March 21, 1977.

UNंIVERSITY OF WISCONSIN

MiLWAUKeE, WI 53201 